\title{
Hereditary Angioedema: a Challenging Diagnosis for the Gastroenterologist
}

\author{
Gabriella Gábos', Dumitru Moldovan²,3, Daniela Dobru1,3 \\ 1 Gastroenterology Department, Mureș County Hospital, Tîrgu Mureș, Romania \\ 2 Allergology-Immunology Department, Mureș County Hospital, Tîrgu Mureș, Romania \\ 3 University of Medicine and Pharmacy, Tîrgu Mureș, Romania
}

\section{CORRESPONDENCE}

\section{Gabriella Gábos}

Str. Gheorghe Marinescu nr. 1

540103 Tîrgu Mures, Romania

Tel:+40 745241974

E-mail: gabriellagabos@yahoo.com

\section{ARTICLE HISTORY}

Received: 8 November, 2016

Accepted: 28 December, 2016
Dumitru Moldovan • Str. Gheorghe Marinescu nr. 1 , 540103 Tîrgu Mures, Romania. Tel: +40 365882588 Daniela Dobru • Str. Gheorghe Marinescu nr. 38 540139 Tîrgu Mures, Romania. Tel: +40 265215551

\begin{abstract}
Hereditary angioedema (HAE) caused by a deficiency of C1 esterase inhibitor enzyme (C1-INH) is a very rare, autosomal dominantly inherited genetic disorder, characterized by recurrent peripheral angioedema, painful abdominal attacks and episodes of laryngeal edema. Abdominal attacks are frequent symptoms in adult HAE patients, occurring in more than $90 \%$ of the cases. Angioedema in the bowel or abdomen can occur in the absence of cutaneous manifestations and may be easily misdiagnosed unless the clinician has a high degree of awareness to include HAE in the differential diagnosis. Misdiagnosis is associated with inadequate treatments, including unnecessary surgical procedures. Any patient who presents recurrent episodes of swelling should be evaluated for HAE caused by C1-INH deficiency. New therapies could save lives and dramatically improve their quality of life.
\end{abstract}

Keywords: hereditary angioedema, C1-INH deficiency, abdominal attacks

\section{INTRODUCTION}

Hereditary angioedema (HAE) due to $\mathrm{C} 1$ esterase inhibitor enzyme (C1-INH) deficiency is an autosomal dominant-inherited disorder, best known for recurrent swellings of any part of the body, without urticaria. ${ }^{1,2}$ The estimated incidence of HAE in the general population is between 1:10,000 to 1:50,000, with no known race, gender or ethnic predominance. ${ }^{3,4}$

Severe upper airway obstruction can lead to death by asphyxiation, thus, it is essential that attacks are correctly diagnosed and quickly treated. ${ }^{4}$

Due to the fact that symptoms and attack locations are usually unpredictable from one episode to the next, the accurate diagnosis of HAE needs a high degree of clinical suspicion.

This review is aimed at increasing awareness among physicians about HAE due to C1-INH deficiency. This article reviews the clinical manifestation, diagnosis and current management of HAE. The case presentation below highlights the inconsistent course of HAE attacks and an extensive delay in the correct diagnosis. 
Taking into consideration the high risk of morbidity and death, recognizing this rare disorder rapidly is critical.

\section{CASE PRESENTATION}

A 25-year-old woman has suffered, since the age of 7 , from periodic edema attacks, which affected the extremities and caused disfigurement. These attacks were related to minor traumas. At the age of 9 , she began experiencing facial attacks. Medical history also showed that the patient had choking symptoms and swallowing difficulties at the age of 12 , which became more frequent in the subsequent years. The symptoms were intermittent with variable severity and frequency. At the age of 17 , she began experiencing recurrent, self-limited episodes of abdominal attack several times a year that were characterized by crampy periumbilical pain, bloating, nausea, and vomiting. Symptoms diminished gradually over $2-3$ days and were followed by watery bowel movements. These abdominal attacks have occured for many years without any respiratory or cutaneous swelling. At the age of 20, during a dental extraction, she had an upper respiratory tract obstruction with voice changes and difficulty swallowing with a duration of 2 days. When she was asked about family history, she reported that the only relative with similar symptoms was her father, who died most probably due to laryngeal edema. Finally, after 18 years, she was diagnosed with type $1 \mathrm{HAE}$.

The patient agreed to the publication of her data and the institution where the patient had been admitted, approved the publication of the case.

\section{CLINICAL MANIFESTATIONS OF HAE}

HAE is a very uncommon and life-threatening genetic condition characterized by intermittent, self-limiting episodes of edema. Recurrent swelling attacks involve the extremities, abdomen, face, genitalia and upper airways. ${ }^{1,2}$

HAE is marked most frequently by a quantitative or functional insufficiency of C1-INH. ${ }^{3,4}$

Although most attacks are self-limiting, laryngeal edema can be mortal due to asphyxiation. Abdominal and peripheral swellings are painful and debilitating. ${ }^{5}$ Among unrecognized or untreated patients, mortality due to sudden and severe obstruction of the upper airway is significant, being predicted at $30 \%{ }^{6}$

The onset of HAE attacks is variable among patients, it starts most often in the first decade of life and becomes a lifelong, chronic and debilitating disorder. ${ }^{7}$ Angioedema symptoms may worsen around adolescence.7,8 The frequency of HAE attacks is highly variable and unpredictable, ranging from one or few per year to one or more per week. Untreated attacks typically last more than $48-96$ hours. $^{8}$

Due to the fact that many clinicians have a low awareness of HAE, this rare condition is frequently underdiagnosed. The accurate identification of HAE may be delayed for decades ( $>10$ years).,7,9 In Romania, the mean age of start of the symptoms is 11.91 years, the mean age when HAE diagnosis is established is 28.40 years and the mean diagnosis delay is 16.49 years.

Most HAE attacks occur in the skin, usually on the extremities, but also on the face, trunk or genitals. However, many other organs may be affected. ${ }^{2,5}$

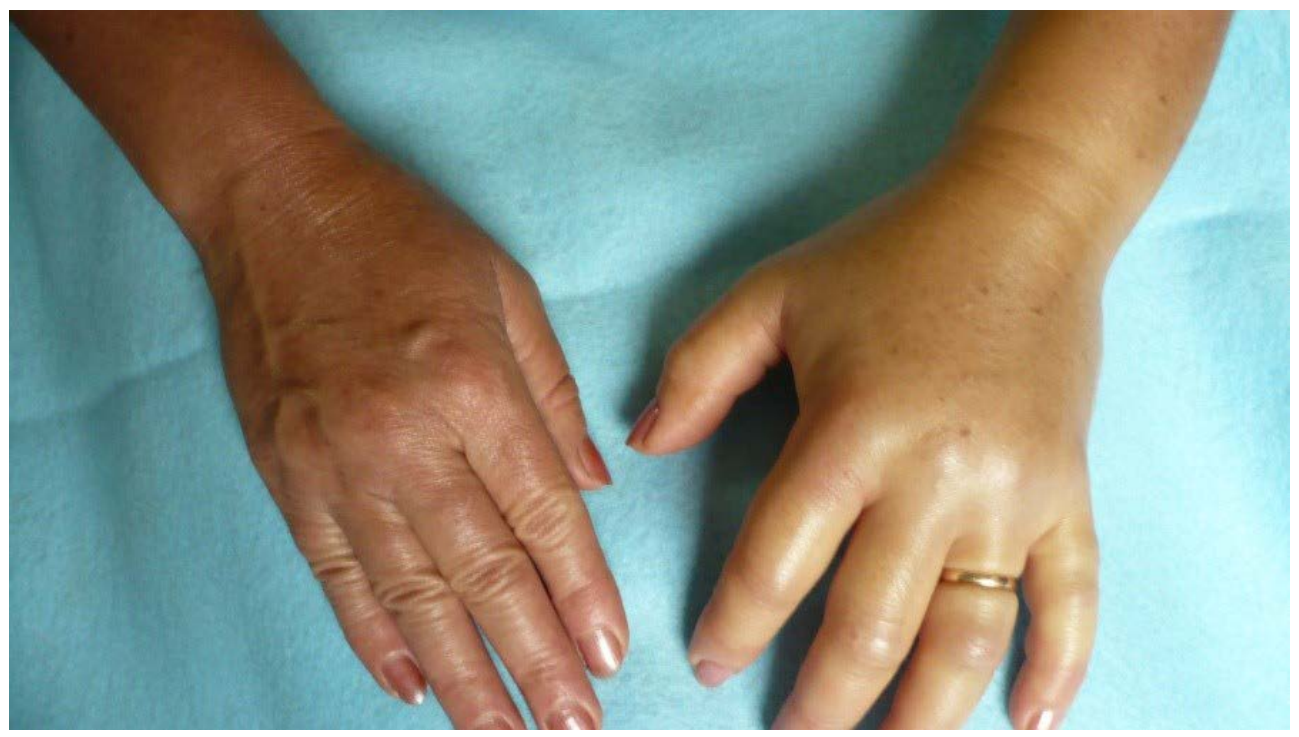

FIGURE 1. Peripheral swelling in HAE attack 


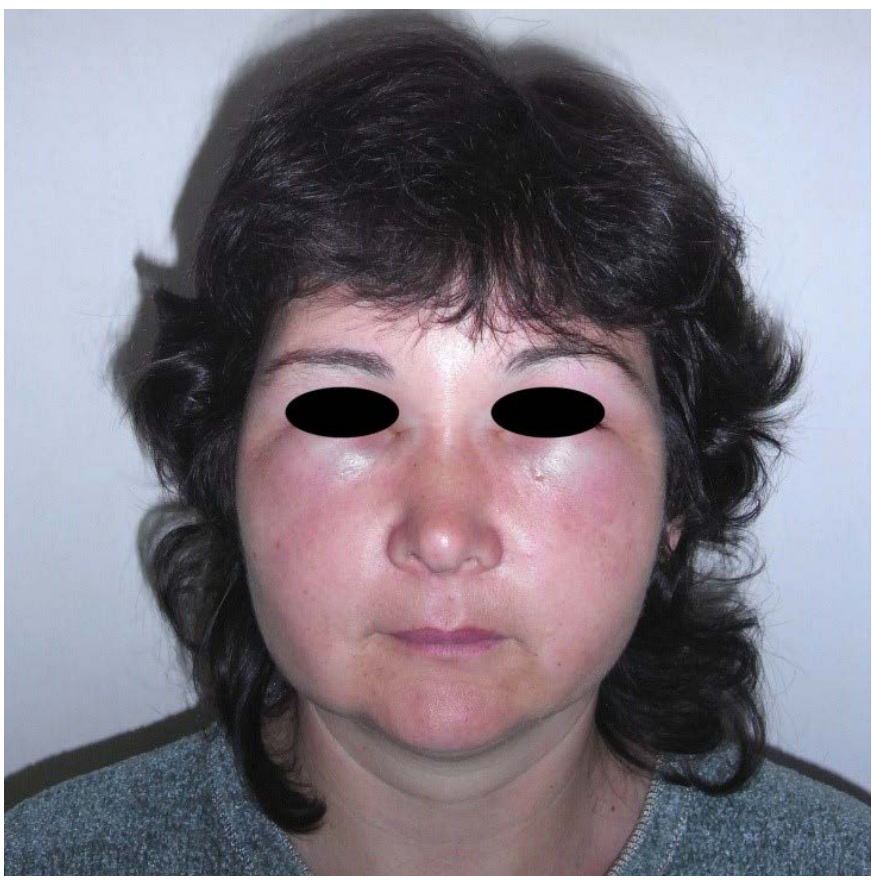

FIGURE 2. Swelling of the face in a patient with type $1 \mathrm{C} 1-\mathrm{INH}$ deficiency

Asymmetric cutaneous swellings are the most characteristic manifestation of HAE, affecting areas of the face, genitals or extremities (Figure 1)., 2,5 Swellings of the skin are typically pale, non-pitting, non-pruritic, painless but uncomfortable. Recurrent episodes of cutaneous swelling unaccompanied by urticaria are the symbol of HAE. Skin attacks typically evolve to the point of disfigurement (Figure 2). ${ }^{5}$

Gastrointestinal tract involvement is important and one of the most common clinical features of HAE.10,11 Abdominal symptoms appear in more than $93 \%$ of the adult HAE

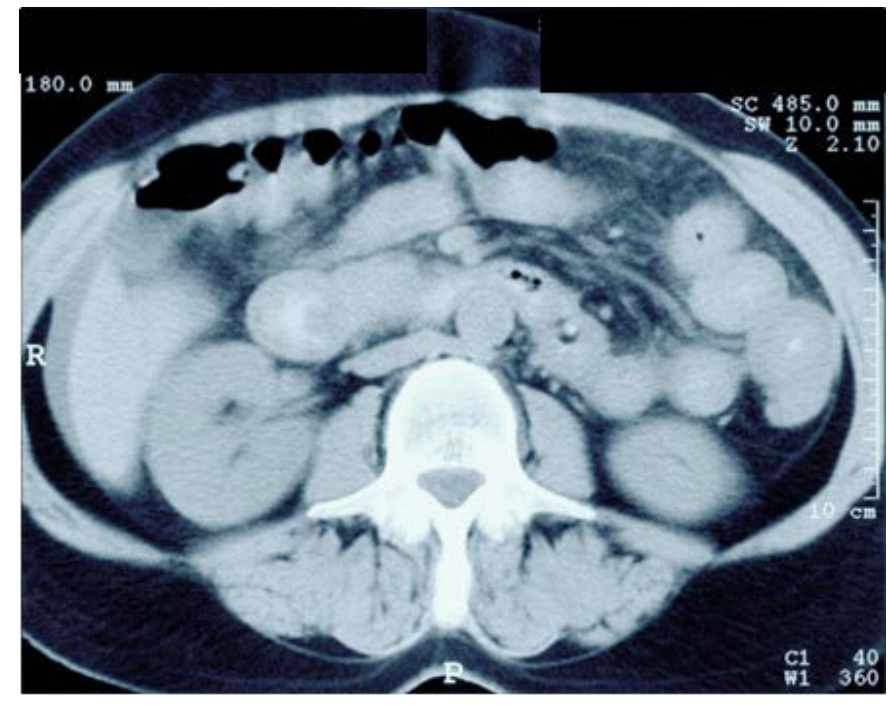

FIGURE 3. Abdominal CT showing multiple sites edema of the bowel associated with an HAE abdominal attack

patients and may be the first and only expression of the disorder. Abdominal pain experienced by HAE patients can be present acutely, or may be intermittent and chronic. ${ }^{12,13}$ The edema of the bowel wall may lead to nausea, vomiting, diarrhea, abdominal pain varying severity, and, in severe cases, fainting due to hypovolemia. ${ }^{14-16}$

The manifestation of an abdominal HAE attack can mimic acute abdominal emergencies (appendicitis, gastroenteritis, cholecystitis, pancreatitis, ischemic bowel) and often leads to a delay in diagnosis, unnecessary invasive surgical procedures or inappropriate pharmacologic treatments. ${ }^{17}$ The difficulty of distinguishing this disease from surgical emergencies remains even after the proper diagnosis of HAE is established. ${ }^{17-19}$

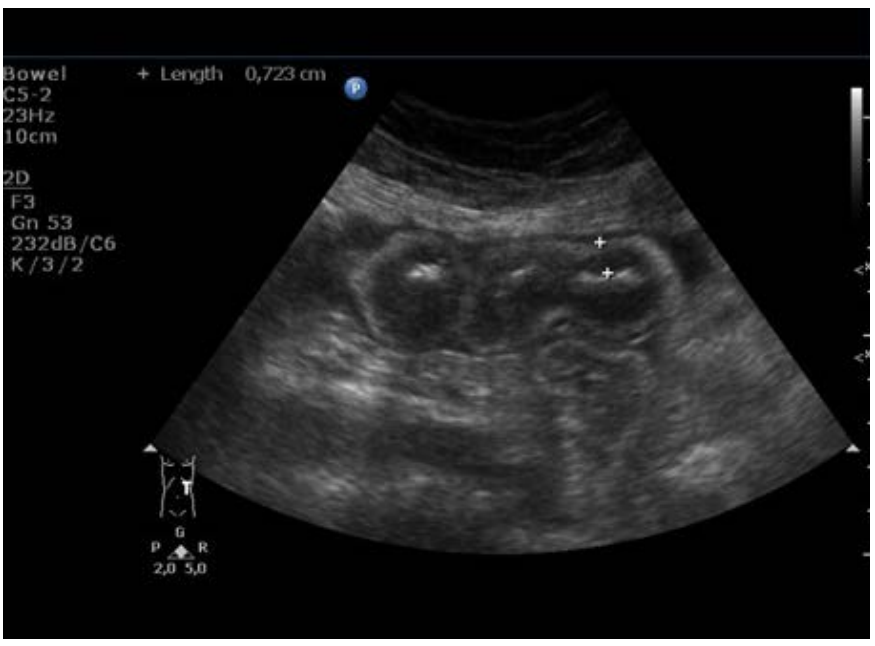

FIGURE 4. Sonographic evidence of bowel thickening

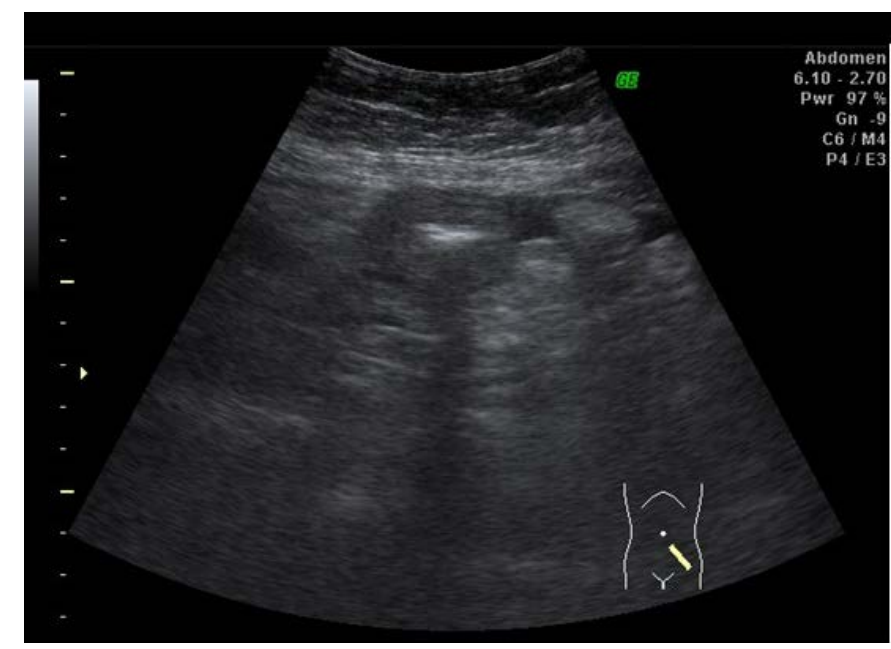

FIGURE 5. Sonographic evidence of ascites between bowels 
Various diagnostic methods were used to confirm the presence of bowel edema and ascites during attacks, hallmarks of the abdominal submucous extravasation of fluid due to bradykinin release (Figures 3-5).18,19

Facial edema is complicated in one-third of the attacks with upper respiratory tract obstruction., ${ }^{2,6}$ The involvement of the larynx by angioedema can result in varying degrees of respiratory distress and even death from suffocation. Clinical manifestations of an upper airway obstruction consist of change in voice quality or hoarseness, difficulty swallowing, shortness of breath, wheezing. ${ }^{5} \mathrm{La}-$ ryngeal edema can advance from mild discomfort to asphyxiation, requiring intubation and/or tracheotomy.5,6 Triggers of laryngeal attacks in HAE patients include dental procedures, upper endoscopy or endotraheal intubation. ${ }^{6}$

Prodromal signs may begin up to several days before an attack and include burning, itching, severe fatigue, myalgia, arthralgia, abdominal cramps and discomfort at the site of attack. ${ }^{20}$ The most characteristic prodromal symptom is erythema marginatum. ${ }^{5,20}$ This rash has serpiginous borders, which are slightly raised, typically involving the trunk and inner surfaces of arms and legs, sparing the face..$^{20}$ Prodromal symptoms usually begin within 24 hours before an attack. Most patients are able to predict the onset of an attack. 2,20,21

A number of possible attack triggers have been proposed in HAE, including any type of trauma, medical, surgical, or dental procedures, psychological stress, infection, hormonal changes, intake of estrogen-containing drugs or angiotensin-converting enzyme inhibitors or prolonged repetitive activity. However, many attacks occur without an obvious trigger, and the same trigger may not always provoke an attack in a specific individual. ${ }^{2}$

HAE can cause substantial physical and emotional distress, limitations in professional or educational achievement, financial hardship, and poor quality of life.,9

\section{DIAGNOSIS}

Insufficient awareness of HAE among physicians can lead to a delay in diagnosis. ${ }^{4} \mathrm{~A}$ history of recurrent attacks of skin edema or abdominal pain starting during childhood or adolescence and refractory to treatment with antihistamines or epinephrine should lead to suspicion of HAE. ${ }^{5}$

A report of similarly affected family members is another important diagnostic clue, although $25 \%$ of patients have de novo mutations and no family history of HAE. 1,5

Idiopathic histaminergic acquired angioedema, idiopathic non-histaminergic acquired angioedema, acquired angioedema related to angiotensin-converting enzyme inhibitors, acquired angioedema with C1-INH deficiency, HAE with C1-INH deficiency, HAE with FXII mutations and HAE of unknown origin make up the differential diagnosis of this disease. ${ }^{3,5}$

Recurrent abdominal crampy pain, vomiting and diarhea may mimic a surgical emergency. ${ }^{21-23}$ When these symptoms are associated with peripheral angioedema without urticaria and upper respiratory obstruction, it is strongly recommended to check the C1-INH activity level and ask the opinion of an angioedema expert. ${ }^{5}$

Laboratory analyses are commonly used for the diagnoses of HAE. Measurement of complement parameters (C4, C1-INH concentration and function, C1q) is suggested. Antigenic and functional C1-INH levels can differentiate type I (antigenic and functional C1-INH levels are below normal levels) from type II (normal or increased levels of C1-INH, which is dysfunctional). A third type of HAE is very rare and remains poorly understood. It occurs mostly in women, it is related to hormone levels in the body and is associated with normal C1-INH protein. ${ }^{1,5}$

\section{PATHOPHYSIOLOGY OF HAE}

The main function of C1-INH is to regulate the activation of the complement, contact, coagulation and fibrinolytic system. ${ }^{5} \mathrm{C} 1-\mathrm{INH}$ deficiency results in excess production of vasoactive mediators such as bradykinin, the primary cause of HAE symptoms.

Local bradykinin generation results in increased vascular permeability and movement of intravascular fluid into the interstitial space, resulting in clinical symptoms of angioedema in a period ranging from minutes to hours. ${ }^{5,8,24}$

\section{MANAGEMENT OF HAE}

The early diagnosis of HAE is a critical step for its ideal management. ${ }^{25}$ The aim of HAE treatment is to decrease morbidity and avoid mortality. Various new drugs are accessible, with differences from country to country. International consensus documents guide the management of HAE due to C1-INH deficiency. ${ }^{25}$

The contemporary therapeutic management of HAE is complex and it is divided into three phases: treatment of acute attacks or on-demand therapy, short-term prophylaxis and long-term prophylaxis..$^{25,26}$

Administration of plasma-derived $\mathrm{C} 1$ inhibitors, recombinant $\mathrm{C} 1$ inhibitor, plasma kallikrein inhibitor, bradykinin $\mathrm{B} 2$ receptor antagonist are effective for the treatment of acute episodes of angioedema caused by C1-INH 
deficiency. ${ }^{25}$ If first-line medication in acute angioedema attacks is not available, 2-3 units of fresh frozen plasma is also useful. ${ }^{26}$

Epinephrine, antihistamines and corticosteroids are not proven to be effective in HAE. ${ }^{26}$

Pain relief is also useful to manage patients suffering from severe abdominal attacks. In progressive, severe upper airway obstruction intubation or tracheotomy may be necessary and life-saving. ${ }^{27}$

Short-term prophylaxis is typically administered before medical maneuvers, particularly in extensive dental work. ${ }^{28}$ Current international guidance and standards recommend the use of C1-INH concentrate or attenuated androgens for preoperative prophylaxis. Attenuated androgens are generally administered 5 days before and 2 days after the procedure..$^{29}$

C1-INH concentrate, attenuated androgens (danazol, stanazolol, oxandrolone) and antifibrinolytic agents are effective in long-term prophylaxis, to decrease the occurrence of HAE episodes. ${ }^{30}$ Attenuated androgens are mostly administered in patients with frequent and severe attacks of HAE. Because the risk of adverse effects (weight gain, reduced libido, anxiety, headache, increased hair growth, hepatotoxicity, liver adenomas) is high, close control of patients is necessary. ${ }^{30}$

\section{CONCLUSIONS}

HAE is often misdiagnosed and poorly managed, because of the lack of sufficient knowledge by the treating physician. There is still a lot of work to do, making hereditary angioedema more popular among different medical specialties: general practitioners, internal medicine specialists, allergists, dermatologists, gastroenterologists, ENT specialists.

Gastroenterologists and other physicians should add intestinal angioedema to their list of potential causes of unexplained abdominal pain and ascites, and ask for C1-INH. Angioedema in the bowel or abdomen can occur in the absence of cutaneous manifestations and may be easily misdiagnosed unless the clinician has a high degree of awareness to include HAE in the differential diagnosis. Timely diagnosis of acute abdominal attacks is essential to prevent a history of unnecessary surgical procedures (laparoscopy or laparotomy). The importance of accurate diagnosis of HAE is paramount in order to determine the most effective treatment options.

\section{CONFLICT OF INTEREST}

Nothing to declare.

\section{REFERENCES}

1. Cicardi M, Bork K, Caballero T, et al. Evidence based recommendations for the therapeutic management of angioedema owing to hereditary $\mathrm{C} 1$ inhibitor deficiency: consensus report of an International Working Group. Allergy. 2012;67:147-157.

2. Bork K, Meng G, Staubach P, Hardt J. Hereditary angioedema: new findings concerning symptoms, affected organs, and corse. The Am J of Med. 2006;119:267-274.

3. Gompels MM, Lock RJ, Abinum M, et al. C1 inhibitor deficiency: consensus document. Clin and Exper Immun. 2005;139:379-394.

4. Zanichelli A, Magerl M, Longhurst H, Fabien V, Maurer M. Hereditary angioedema with $\mathrm{C} 1$ inhibitor deficiency: delay in diagnosis in Europe. Allergy Asthma Clin Immunol. 2013;9:29.

5. Longhurst H, Cicardi M. Hereditary angio-oedema. Lancet. 2012;379:474481.

6. Bork K, Ressel N. Sudden upper airway obstruction in patients with hereditary angioedema. Transfus Aphers Sci. 2003;29:235-238.

7. Lumry WR, Castaldo AJ, Vernon MK, Blaustein MB, Wilson DA, Horn PT. The humanistic burden of hereditary angioedema: impact on healthrelated quality of life, productivity, and depression. Allergy Asthma Proc. 2010;31:407-414.

8. Bernstein JA. Update on agioedema: Evaluation, diagnosis, and treatment. Allergy Asthma Proc. 2011;32:408-412.

9. Prior N, Remor E, Traseira CG, et al. Development of a disease-specific quality of life questionnaire for adult patients with hereditary angioedema due to $\mathrm{C} 1$ inhibitor deficiency (HAE-QoL): Spanish multi-centre research project. Health and Quality of Life Outcomes. 2012;10:82.

10. Bork K, Staubach P, Eckardt AJ, et al. Symtoms, course, and complications of abdominal attacks in hereditary angioedema due to $\mathrm{C} 1$ inhibitor deficiency. Am J Gastroenterol. 2006;101:619-627.

11. Nzeako UC, Longhurst HJ. Many faces of angioedema: focus on the diagnosis and the management of abdominal manifestations of hereditary angioedema. Eur J Gastroenterol Hepatol. 2012;24:353-361.

12. Jalaj S, Scolapio JS. Gastrointestinal Manifestations, Diagnosis, and Management of Hereditary Angioedema. J Clin Gastroenterol. 2013;47:817-823.

13. Ali MA, Borum ML. Hereditary angioedema: what the gastroenterologist needs to know. Clin and Exp Gastroenterol. 2014;7:435-445

14. Chen X, Yang YX, Liu YL, Gan HT, Wen ZH. Hereditary angioedema a rare cause of recurrent abdominal pain. Pak J Med Sci. 2014;30:1147-1149.

15. Rubinstein E, Stolz LE, Sheffer A, Stevens C, Bousvaros A. Abdominal attacks and treatment in hereditary angioedema with C1-inhibitor deficiency. BMC Gastroenterol. 2014;14:71.

16. Jesic R, Culafic D, Bonacic-Nikolic B. Hereditary angioedema presenting with recurent ascites. Digestive Diseases and Sciences. 2005;50:24-26.

17. Killedar MM, Malani AS. Hereditary angioedema-presenting as recurrent abdominal pain. Indian J Surg. 2011;73:444-446.

18. Wakisaka M, Shuto $M$, Abe $H$, et al. Computed tomography of gastrointestinal manifestation of hereditary angioedema. Radiat Med. 2008;26:618-621.

19. Riguzzi C, Losonczy L, Teismann N, Herring AA, Nagdev A. Gastrointestinal manifestation of hereditary angioedema diagnosed by ultrasound in the emergency department. West J Emerg Med. 2014;15:816-818.

20. Reshef A, Prematta MJ, Craig TJ. Signs and symptoms preceding acute attacks of hereditary angioedema: results of three recent surveys. Allergy Asthma Proc. 2013;34:261-266.

21. Prematta MJ, Bewtra AK, Levy RJ, et al. Per-attack reporting of prodromal symptoms concurrent with C1-inhibitor treatment of herditary angioedema attacks. Adv Ther. 2012;29:913-922.

22. Gábos G, Pascarenco O, Bara N, Mihaly E, Moldovan D. Atacurile abdominale din angioedemul ereditar: cauză ignorată a durerilor abdominale recurente. Journal of Gastrointestinal and Liver Disease. 2014;23:234-235

23. Gábos G, Mihály E, Bara N, Pascarenco O, Moldovan D. Abdominal recurrent attacks in hereditary angioedema due to $\mathrm{C} 1$ inhibitor deficiency. Journal of the Romanian Society of Allergology and Clinical Immunology. 2015;XII:12-17.

24. Kaplan AP. Enzymatic pathways in the pathogenesis of hereditary angioedema: the role of $\mathrm{C} 1$ inhibitor therapy. J Allergy Clin Immunol. 2010;126:918-925.

25. Bowen T, Cicardi M, Farkas H, et al. 2010 International consensus algorithm for the diagnosis, therapy and management of hereditary angioedema. Allergy Asthma Clin Immunol. 2010;6:24. 
26. Bork K, Davis-Lorton M. Overview of hereditary angioedema caused by C1-inhibitor deficiency: assessment and clinical management. Eur Ann Allergy Clin Immunol. 2013;45:7-16.

27. Bowen T, Cicardi M, Farkas H, Bork K, Kreuz W, et al. Canadian 2003 International Consensus Algorithm For the Diagnosis, Therapy, and Management of Hereditary Angioedema. J Allergy Clin Immunol. 2004:114:629-637.

28. Cicardi M, Aberer W, Banerji A, et al. Classification, diagnosis, and approach to treatment for angioedema: Consensus report from the Hereditary Angioedema International Working Group. Allergy. 2014;69:602-616.

29. Cicardi M, Bellis P, Bertazzoni G, et al. Guidance for diagnosis and treatment of acute angioedema in the emergency department: consensus statement by a panel of Italian experts. Intern Emerg Med. 2014;9:85-92.

30. Longhurst HJ, Tarzi MD, Ashworth F, et al. C1 inhibitor deficiency: 2014 United Kingdom consensus document. Clin and Exper Imm. 2015;180:475483. 